\title{
Impact of Nonstandard Interactions on Neutrino-Nucleon Scattering
}

\author{
D. K. Papoulias and T. S. Kosmas \\ Theoretical Physics Section, University of Ioannina, 45110 Ioannina, Greece \\ Correspondence should be addressed to D. K. Papoulias; dimpap@cc.uoi.gr
}

Received 13 July 2016; Revised 30 September 2016; Accepted 15 November 2016

Academic Editor: Burak Bilki

Copyright ( 2016 D. K. Papoulias and T. S. Kosmas. This is an open access article distributed under the Creative Commons Attribution License, which permits unrestricted use, distribution, and reproduction in any medium, provided the original work is properly cited. The publication of this article was funded by SCOAP ${ }^{3}$.

\begin{abstract}
Nonstandard neutrino-nucleon interaction is formulated and explored within the energy range of quasi-elastic scattering. In particular, the study focuses on the neutral-current elastic (anti)neutrino scattering off nucleons described by the exotic reactions $v_{\alpha}\left(\bar{\nu}_{\alpha}\right)+n \rightarrow v_{\beta}\left(\bar{v}_{\beta}\right)+n$ and $\nu_{\alpha}\left(\bar{\nu}_{\alpha}\right)+p \rightarrow v_{\beta}\left(\bar{\nu}_{\beta}\right)+p$, which provide corrections to the dominant Standard Model processes. In this context, it is shown that the required exotic nucleon form factors may have a significant impact on the relevant cross sections. Besides cross sections, the event rate is expected to be rather sensitive to the magnitude of the lepton-flavour violating parameters resulting in an excess of events. The overlap of nonstandard interactions and strange quark contributions, in the region of few $\mathrm{GeV}$ neutrino energies, is also examined. The formalism is applied for the case of the relevant neutrino-nucleon scattering experiments (LSND, MiniBooNE, etc.) and motivates the notion that such facilities have high potential to probe NSI.
\end{abstract}

\section{Introduction}

Neutrinos are among the most elusive particles in nature and in order to investigate their properties $[1,2]$ various terrestrial detectors have been built [3]. These ghostly particles fill the whole universe and reach Earth coming from the sun (solar neutrinos), from supernova explosions (supernova neutrinos), and from many other celestial objects (e.g., black hole binary stars, active galactic nuclei) [4]. The majority of them pass through Earth and through the nuclear detectors, designed for such purposes, without leaving any trace or signal [5]. This is mostly due to the fact that neutrinos interact very weakly with matter $[6,7]$. For the investigation of neutrino-matter scattering, it is feasible to employ powerful accelerators operating at major laboratories such as Fermilab, J-Park, and CERN. These facilities can produce intensive neutrino beams of which a tiny fraction can be detected by novel detectors placed in the beam line (e.g., COHERENT experiment at Oak Ridge [8], TEXONO experiment in Taiwan [9], $\nu \mathrm{GeN}$ [10] and GEMMA [11] experiments in Russia, and CONNIE project in Brazil $[12,13])$.

On the theoretical side, phenomenological models within and beyond the standard electroweak theory come out with theoretical predictions for many aspects of neutrinos in trying to understand their properties and interactions [14] and propose appropriate neutrino probes for extracting new experimental results [15-17]. Current important areas of research concern the neutrino masses [18], neutrino oscillations $[19,20]$, neutrino electromagnetic properties [21-25], and so forth, their role in the evolution of astrophysical sources such as the sun or supernovae [26-28], their impact on cosmology (e.g., in answering the question of the matterantimatter asymmetry of the universe), and others.

The neutral-current elastic (NCE) and charged-current quasi-elastic (CCQE) scattering of neutrinos with nucleons and nuclei constitute examples of fundamental electroweak interactions within the Standard Model (SM) [29], which, despite their relative simplicity, are presently not well understood. The first attempts of experimentally measuring the cross sections of the latter processes resulted in a discrepancy [30-32] with the predictions of the widely used relativistic Fermi gas (RFG) model [33-37]. There has been much effort towards quantifying this disagreement (between theory and experiment), mainly in terms of the nucleon electromagnetic form factors at intermediate energies $[38,39]$, while other 


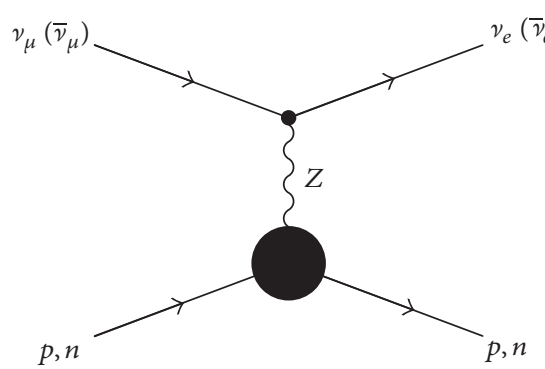

(a)

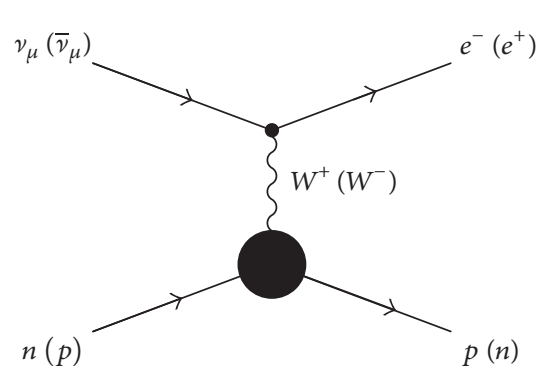

(b)

FIGURE 1: Diagrams of nonstandard neutrino-nucleon interactions for neutral-current (a) and charged-current (b) processes.

works focus on the study of the potential contribution of the strange components of the hadronic current [40-43].

Over a decade ago, an anomalous excess of events has been reported by the LSND experiment in searching for $\nu_{\mu} \rightarrow$ $\nu_{e}$ oscillations [44]. Recently, in the MiniBooNE neutrinonucleon scattering experiment, an unexplained excess of electron-like events, $\Delta N=128.8 \pm 20.4 \pm 38.3$, has been observed in the reconstructed neutrino energy range $200 \leq$ $E_{v} \leq 475 \mathrm{MeV}$ [45-49]. To interpret these data, some authors [50] argued that the RFG model is insufficient to accurately describe the neutrino-nucleon interaction in nuclei embedded in dense media [50], while other authors paid special attention to the final state interaction (FSI) effects [51-53]. Furthermore, this anomaly has triggered the intense theoretical interest and towards its explanation several models have been proposed $[54,55]$ including also those addressing heavy Dirac or Majorana neutrino decay $[56,57]$, the existence of sterile neutrinos [58], and others.

Historically, nucleons and nuclear systems have been extensively employed $[59,60]$ as microlaboratories for exploring open neutrino properties through charged- [61] and neutral-current interaction processes [62]. The latter involve both the vector and the axial vector components of the weak interactions $[63,64]$. Thus, such probes are helpful for investigating the fundamental interactions of neutrinos with other elementary particles at low, intermediate, and high energies [65]. For the case of neutral-current coherent elastic neutrino scattering off complex nuclei, a detailed analysis focusing on possible alterations of the expected event rates due to the existence of nonstandard interactions (NSI) was performed in our previous works [66-68].

Motivated by the latter, in this paper, we explore the possibility of probing exotic neutrino processes in the relevant experiments. Thus, within the framework of NSI [69] (for a review, see $[70,71])$, we consider the NCE scattering of the following neutrino-nucleon reactions:

$$
\begin{aligned}
& v_{\alpha}\left(\bar{\nu}_{\alpha}\right)+n \longrightarrow v_{\beta}\left(\bar{v}_{\beta}\right)+n, \\
& v_{\alpha}\left(\bar{v}_{\alpha}\right)+p \longrightarrow v_{\beta}\left(\bar{v}_{\beta}\right)+p,
\end{aligned}
$$

where $\alpha, \beta=\{e, \mu, \tau\}$ denote the neutrino flavour. Specifically, for the case of the MiniBooNE processes, we concentrate on the channels where $\alpha=\mu$ and $\beta=e$ (for NSI scattering involving tau neutrinos, see $[72,73])$. In the present study, the magnitude of the proposed novel interactions is given in terms of the adopted NSI nucleon form factors as functions of the four-momentum transfer. In our effort to explore potential NSI neutrino-nucleon interactions, as a first step, we focus only on the NSI Feynman diagrams depicted in Figure 1 where, for completeness, the corresponding diagrams involving charged-current (CC) processes are also included.

\section{Neutral-Current Nonstandard Neutrino-Nucleon Interactions}

In the present work, the assumed NSI operators are effective four-fermion operators of the form [70]

$$
\mathcal{O}=\left(\bar{f}_{1} \gamma^{\mu} P f_{2}\right)\left(\bar{f}_{3} \gamma_{\mu} P f_{4}\right)+\text { h.c., }
$$

with $f_{i}, i=1,2,3,4$ being the SM fermion fields and $P=$ $\{L, R\}$ denoting left- and right-handed projectors. Specifically, for neutral currents, one has neutrino-induced NSI with matter of the form [69]

$$
\mathcal{O}_{\alpha \beta}^{f P}=\left(\bar{\nu}_{\alpha} \gamma^{\mu} L v_{\beta}\right)\left(\bar{f} \gamma_{\mu} P f\right)+\text { h.c., }
$$

with $f$ denoting a first-generation quark $q=\{u, d\}$.

2.1. Neutrino-Nucleon Cross Sections within and beyond the $S M$. The calculations of the neutrino-nucleon cross sections start by writing down the nucleon matrix elements of processes (1) and (2) in the usual $V-A$ form, as

$$
\begin{aligned}
\mathscr{M} & =\frac{i G_{F}}{2 \sqrt{2}} j_{\mu}\left\langle\mathcal{N}\left|J_{Z}^{\mu}\right| \mathscr{N}\right\rangle \\
& =\frac{i G_{F}}{2 \sqrt{2}} \bar{\nu}_{\alpha} \gamma_{\mu}\left(1-\gamma_{5}\right) \nu_{\beta}\left\langle\mathcal{N}\left|J_{Z}^{\mu}\right| \mathcal{N}\right\rangle,
\end{aligned}
$$

where $j_{\mu}$ denotes the leptonic neutral current, $G_{F}$ is the Fermi coupling constant, and $|\mathcal{N}\rangle$ represents the nucleon wavefunction. In the latter expression, $\left\langle\mathcal{N}\left|J_{Z}^{\mu}\right| \mathcal{N}\right\rangle$ is the hadronic matrix element that is (after neglecting the second-class 
currents and the contribution of pseudoscalar component) expressed in terms of the known nucleon form factors as [7]

$$
\begin{aligned}
& \left\langle\mathcal{N}\left|J_{Z}^{\mu}\right| \mathcal{N}\right\rangle=\langle\mathcal{N}| F_{1}^{\mathrm{NC}: p(n)}\left(Q^{2}\right) \\
& +F_{2}^{\mathrm{NC}: p(n)}\left(Q^{2}\right) \frac{i \sigma^{\mu \nu} q_{\nu}}{2 m_{\mathcal{N}}}+F_{A}^{\mathrm{NC}: p(n)}\left(Q^{2}\right) \gamma^{\mu} \gamma^{5}|\mathcal{N}\rangle .
\end{aligned}
$$

In (6), $F_{1}^{\mathrm{NC}: p^{(n)}}\left(Q^{2}\right), F_{2}^{\mathrm{NC}: p(n)}\left(Q^{2}\right)$, and $F_{A}^{\mathrm{NC}: p(n)}\left(Q^{2}\right)$ stand for the Dirac, Pauli, and axial vector weak neutral-current form factors, respectively, for protons $(p)$ or neutrons $(n)$ [1].

Relying on the above nucleon matrix elements, within the relativistic Fermi gas (RFG) model, the SM differential cross section of reactions (1) and (2) for incoming (anti)neutrino energy $E_{v}$ has been written as [29]

$$
\frac{d \sigma}{d Q^{2}}=\frac{G_{F}^{2} Q^{2}}{2 \pi E_{v}^{2}}\left[A\left(Q^{2}\right) \pm B\left(Q^{2}\right) W+C\left(Q^{2}\right) W^{2}\right]
$$

In the above expression, the plus (minus) sign accounts for neutrino (antineutrino) scattering while the momentumdependent function, $W\left(Q^{2}\right)$, reads [41]

$$
W=\frac{4 E_{v}}{m_{\mathcal{N}}}-\frac{Q^{2}}{m_{\mathcal{N}}^{2}},
$$

where the four-momentum transfer is defined in terms of the nucleon recoil energy $T_{\mathcal{N}}$, as

$$
q^{2}=q_{\mu} q^{\mu}=-Q^{2}=-2 m_{\mathscr{N}} T_{\mathcal{N}}
$$

For the nucleon mass, $m_{\mathcal{N}}$, we assume the value $m_{p} \approx m_{n}=$ $m_{\mathcal{N}}=0.938 \mathrm{GeV}$.

Before proceeding to the cross sections calculations, for the reader's convenience, we provide below some significant details on the aforementioned expressions $A, B$, and $C$ that depend on the form factors $F_{i}^{\mathrm{NC}: p(n)}, i=1,2, A$. The functions $A\left(Q^{2}\right), B\left(Q^{2}\right)$, and $C\left(Q^{2}\right)$ are defined as [42]

$$
\begin{aligned}
& A\left(Q^{2}\right)=\frac{1}{4}\left\{\left(F_{A}^{\mathrm{NC}: p(n)}\right)^{2}(1+\tau)\right. \\
& -\left[\left(F_{1}^{\mathrm{NC}: p(n)}\right)^{2}-\tau\left(F_{2}^{\mathrm{NC}: p(n)}\right)^{2}\right](1-\tau) \\
& \left.+4 \tau F_{1}^{\mathrm{NC}: p(n)} F_{2}^{\mathrm{NC}: p(n)}\right\}, \\
& B\left(Q^{2}\right)=-\frac{1}{4} F_{A}^{\mathrm{NC}: p(n)}\left(F_{1}^{\mathrm{NC}: p(n)}+F_{2}^{\mathrm{NC}: p(n)}\right), \\
& C\left(Q^{2}\right)=\frac{m_{\mathcal{N}}^{2}}{16 Q^{2}} l\left[\left(F_{A}^{\mathrm{NC}: p(n)}\right)^{2}+\left(F_{1}^{\mathrm{NC}: p(n)}\right)^{2}\right. \\
& \left.+\tau\left(F_{2}^{\mathrm{NC}: p(n)}\right)^{2}\right],
\end{aligned}
$$

where their explicit $Q^{2}$ dependence has been suppressed and $\tau=Q^{2} / 4 m_{\mathcal{N}}^{2}$.
In principle, the electromagnetic Dirac and Pauli form factors are written in terms of the well-known electric (E) and magnetic (M) form factors as follows [1]:

$$
\begin{aligned}
& F_{1}^{\mathrm{EM}: p(n)}=\frac{G_{\mathrm{E}}^{p^{(n)}}\left(Q^{2}\right)+\tau G_{\mathrm{M}}^{p(n)}\left(Q^{2}\right)}{1+\tau}, \\
& F_{2}^{\mathrm{EM}: p(n)}=\frac{G_{\mathrm{M}}^{p^{(n)}}\left(Q^{2}\right)-\tau G_{\mathrm{E}}^{p(n)}\left(Q^{2}\right)}{1+\tau} .
\end{aligned}
$$

In this work, the magnetic form factors are parametrised as [39]

$$
\frac{G_{\mathrm{M}}^{p(n)}}{\mu_{p(n)}}=\frac{1+a_{p(n), 1}^{\mathrm{M}} \tau}{1+b_{p(n), 1}^{\mathrm{M}} \tau+b_{p(n), 2}^{\mathrm{M}} \tau^{2}+b_{p(n), 3}^{\mathrm{M}} \tau^{3}}
$$

where $\mu_{p(n)}$ denotes the proton (neutron) magnetic moment. The proton electric form factor in a similar manner can be cast in the form [26]

$$
G_{\mathrm{E}}^{p(n)}=\frac{1+a_{p(n), 1}^{\mathrm{E}} \tau}{1+b_{p(n), 1}^{\mathrm{E}} \tau+b_{p(n), 2}^{\mathrm{E}} \tau^{2}+b_{p(n), 3}^{\mathrm{E}} \tau^{3}},
$$

(for the fit parameters, $a_{p(n), 1}^{\mathrm{M}(\mathrm{E})}$ and $b_{p(n), j}^{\mathrm{M}(\mathrm{E})}, j=1,2,3$, see [39]). The electric neutron form factor, $G_{\mathrm{E}}^{n}$, is expressed through the Galster-like parametrisation, as

$$
G_{\mathrm{E}}^{n}\left(Q^{2}\right)=\frac{\lambda_{1} \tau}{1+\lambda_{2} \tau} G_{D}\left(Q^{2}\right)
$$

with $\lambda_{1}=1.68$ and $\lambda_{2}=3.63$.

2.2. NSI Nucleon Form Factors. As it is well known, within the SM, the weak NC Dirac and Pauli form factors are written in terms of the electromagnetic current form factors $F_{i}^{\mathrm{EM}}, i=$ 1,2 (assuming the conserved vector current (CVC) theory) [1]. In the present work, we furthermore consider additional contributions originating from NSI that enter through the vector-type form factors $\varepsilon_{\mu e}^{q V}\left(Q^{2}\right)$. In our parametrisation, the latter are written in terms of the fundamental NSI neutrinoquark couplings $\epsilon_{\mu e}^{u V}\left(\epsilon_{\mu e}^{d V}\right)$ for $u(d)$ quarks discussed in [6668 ], and they take the form

$$
\begin{aligned}
& \varepsilon_{\mu e}^{p V}\left(Q^{2}\right)=\left(2 \epsilon_{\mu e}^{u V}+\epsilon_{\mu e}^{d V}\right) G_{D}\left(Q^{2}\right), \\
& \varepsilon_{\mu e}^{n V}\left(Q^{2}\right)=\left(\epsilon_{\mu e}^{u V}+2 \epsilon_{\mu e}^{d V}\right) G_{D}\left(Q^{2}\right) .
\end{aligned}
$$

In the spirit of previous studies which consider the strangeness of the nucleon [41, 42], the above NSI form factors may have the same momentum dependence as those of the SM ones. Thus, the function $G_{D}\left(Q^{2}\right)$ is assumed to be of dipole type:

$$
G_{D}=\left(1+\frac{Q^{2}}{M_{V}^{2}}\right)^{-2}
$$

(for the vector mass, a commonly used value is $M_{V}=0.843$ $\mathrm{GeV})$. A dipole approximation for $G_{D}\left(Q^{2}\right)$, apart from 


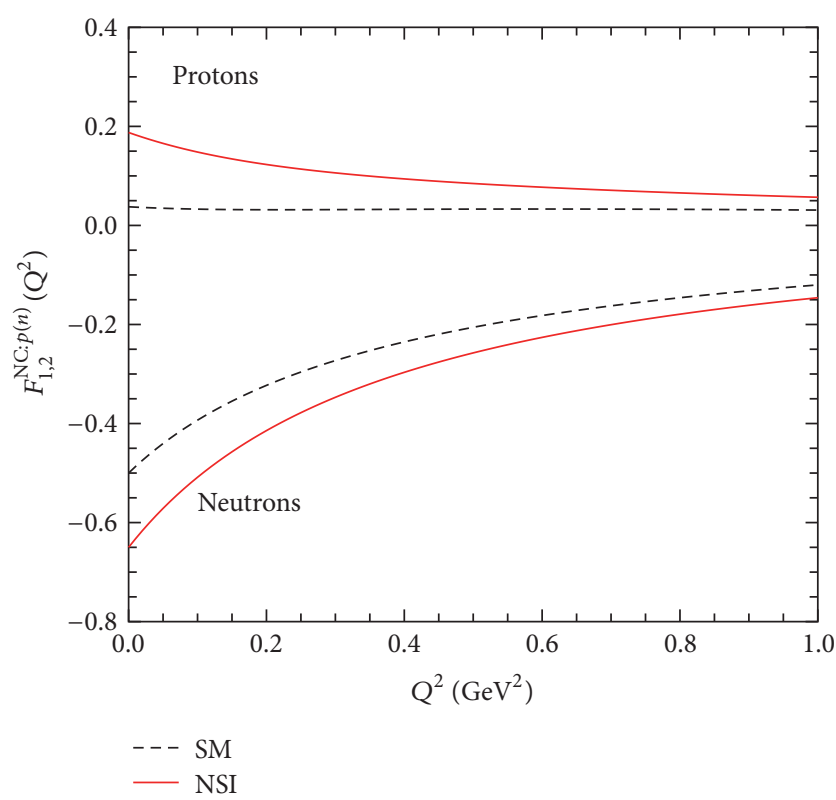

FIgure 2: Comparison of the SM and NSI nucleon form factors employed in the present study (for details, see the text).

providing the appropriate momentum dependence, ensures also that the event rate coming out of NSI has the correct behaviour at high energies [40].

Then, the weak neutral-current nucleon form factors for protons (plus sign) and neutrons (minus sign) employed in our present calculations read

$$
\begin{aligned}
F_{1,2}^{\mathrm{NC}: p(n)}\left(Q^{2}\right)= & \frac{\tau_{3}}{2}\left[F_{1,2}^{\mathrm{EM}: p}\left(Q^{2}\right)-F_{1,2}^{\mathrm{EM}: n}\left(Q^{2}\right)\right] \\
& -2 \sin ^{2} \theta_{W} F_{1,2}^{\mathrm{EM}: p(n)}\left(Q^{2}\right) \\
& -\frac{1}{2} F_{1,2}^{\mathrm{s} p(n)}\left(Q^{2}\right)+\tau_{3} \varepsilon_{\mu e}^{p(n) V}\left(Q^{2}\right) .
\end{aligned}
$$

In the latter expression, the isoscalar form factors $F_{1,2}^{s: p(n)}$ account for potential contributions to the electric charge and the magnetic moment of the nucleon due to the presence of strange quarks (in our convention, the isospin index $\tau_{3}$ is +1 for proton and -1 for neutron scattering). However, throughout our calculations, on the basis of the recent results from the HAPPEX experiment [43], we take $F_{1,2}^{\mathrm{s}: p^{(n)}}=0$ (see also [52]). Note that, for low momentum transfer, the form factors discussed in $[66,67]$ are recovered. The effect of NSI on the form factors is illustrated graphically in Figure 2, where typical values have been adopted for the NSI parameters; that is, $\epsilon_{\mu e}^{u V}=\epsilon_{\mu e}^{d V}=0.05$ (apparently strange quark contributions have no impact in this case). Within the present formalism, the new nucleon form factors are rather sensitive to NSI, even for small values of the fundamental model parameters, especially for low momentum transfer.
For the case of the axial form factor $F_{A}^{\mathrm{NC}: p(n)}$, we correspondingly employed [61]

$$
F_{A}^{\mathrm{NC}: p(n)}\left(Q^{2}\right)=\frac{1}{2}\left(\tau_{3} g_{A}+g_{A}^{\mathrm{s}}\right)\left(1+\frac{Q^{2}}{M_{A}^{2}}\right)^{-2} \text {. }
$$

Here, we used the static axial vector coupling, $g_{A}=-1.267$ (it is determined usually through neutron beta decay). For the strange quark contribution to the nucleon spin, we adopt the static value $2 F_{A}^{s}(0)=g_{A}^{s} \pm 0.07$ with $g_{A}^{s}=-0.15$, while for the axial mass we take $M_{A}=1.049 \mathrm{GeV}$ (i.e., fit II of [41]). As has been recently discussed in [54], this set of values is fully compatible with the MiniBooNE data, even though a large value of $M_{A}=1.35 \mathrm{GeV}$ was reported in [47]. Furthermore, for simplicity, potential axial NSI form factors are neglected.

\section{Results and Discussion}

At first, we calculate the differential cross sections of (7) for the SM $(\alpha=\beta)$ weak NC elastic scattering processes (1) and (2) as well as for the NSI ones $(\alpha \neq \beta)$, based on (15)(18) (neglecting potential strange quark contributions, i.e., $F_{1,2}^{s: p(n)}=0$ and $\left.g_{A}^{s}=0\right)$. The corresponding results are demonstrated in Figure 3 for $v p \rightarrow v p$ scattering (a) and $\nu n \rightarrow \nu n$ scattering (b). For the sake of comparison, the bands of axial vector strange quark contributions calculated within $g_{A}^{\mathrm{s}} \pm 0.07$ and those of NSI contributions within the range $\epsilon_{\mu e}^{q V}=(-0.05,0.05)$ with $q=\{u, d\}$ are also depicted. One sees that the resulting strange quark contributions indicate almost equal cross sections for proton and neutron scattering, while the presence of NSI leads to an enhancement of the cross sections for both $\nu p \rightarrow \nu p$ and $\nu n \rightarrow v n$ scattering channels, which becomes more important at lower energies.

From the perspective of experimental physics, it is crucial to reduce most of the background as well as beam related and systematic uncertainties. Therefore, a rather advantageous way towards determining the strange or NSI parameters is to perform measurements of the ratio of the NCE cross sections:

$$
R=\frac{d \sigma_{p} / d Q^{2}}{d \sigma_{n} / d Q^{2}}
$$

In this context, Figure 4 illustrates a comparison of the obtained bands for the ratio $R$ assuming neutrino-nucleon scattering in the presence of strange quarks or potential NSI. One notices that $R$ varies between 0.75 and 1.20 when axial vector strange quark contributions are taken into account, while for the case of NSI the ratio is significantly lower; that is, it lies between 0.55 and 0.8 . It is furthermore shown that, unlike the strange quark case, the SM result for $R$ lies within the predicted NSI band.

Focusing on the relevant experiments, in order to perform reliable calculations, important effects that originate from the Pauli principle must be taken into account (Fermi motion of the initial nucleons). Specifically, for the case of nucleons bound in nuclear matter, within the Fermi gas model, it is adequate to multiply the free cross section given in (7) with a suppression factor $S\left(Q^{2}\right)$. The latter accounts for the 


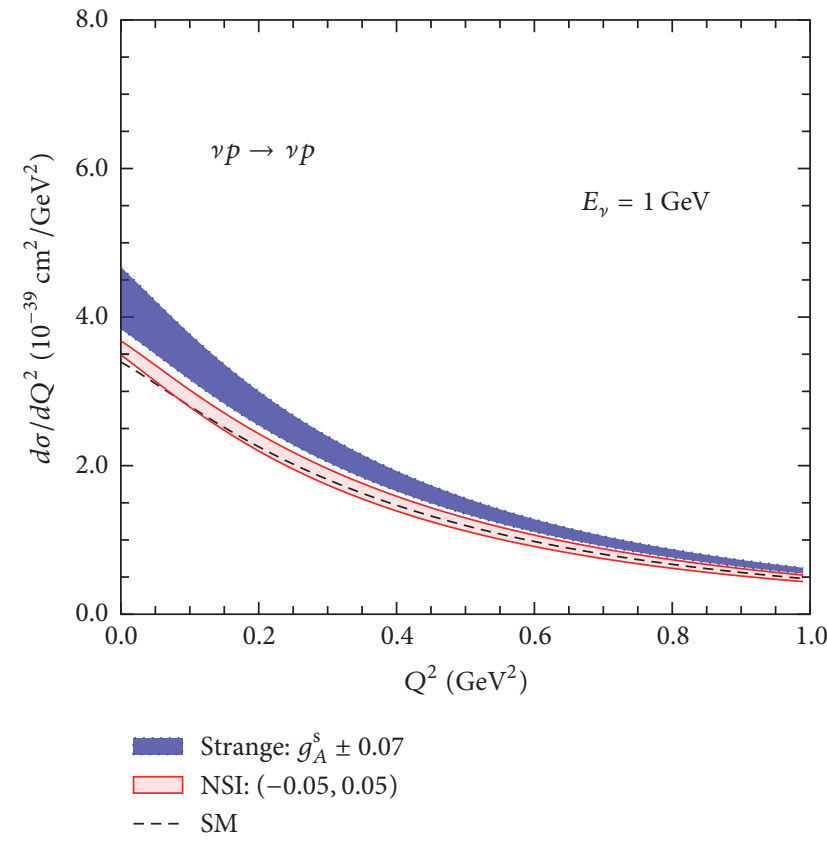

(a)

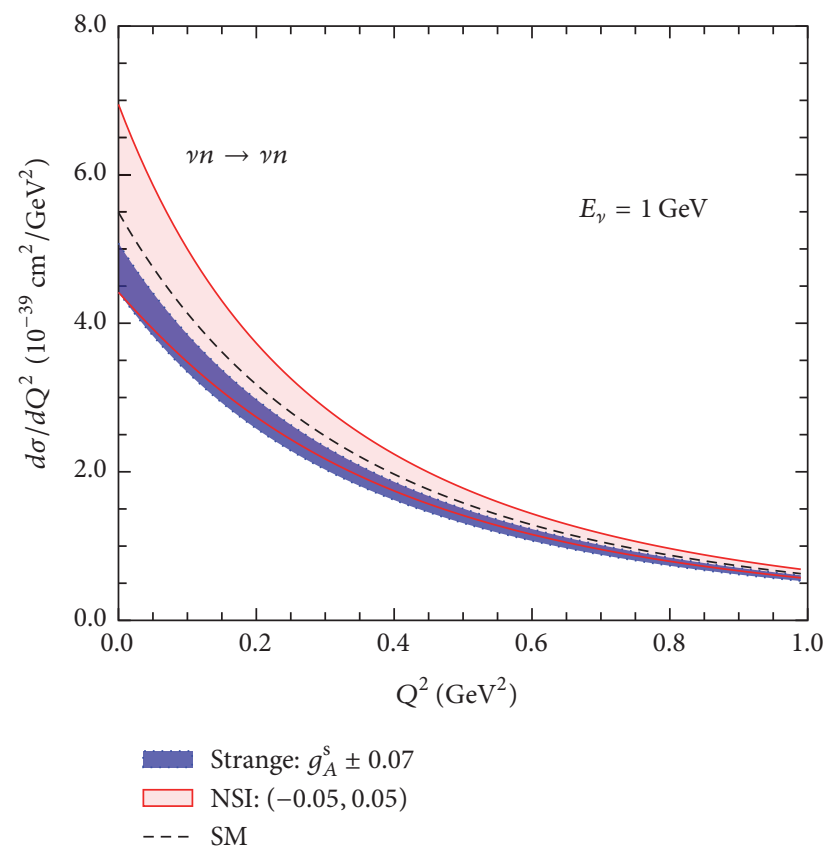

(b)

FIGURE 3: Differential cross section with respect to the momentum transfer for SM, strange quark, and NSI, for $v p \rightarrow \nu p($ a) and $\nu n \rightarrow \nu n$ scattering (b). For details, see the text.

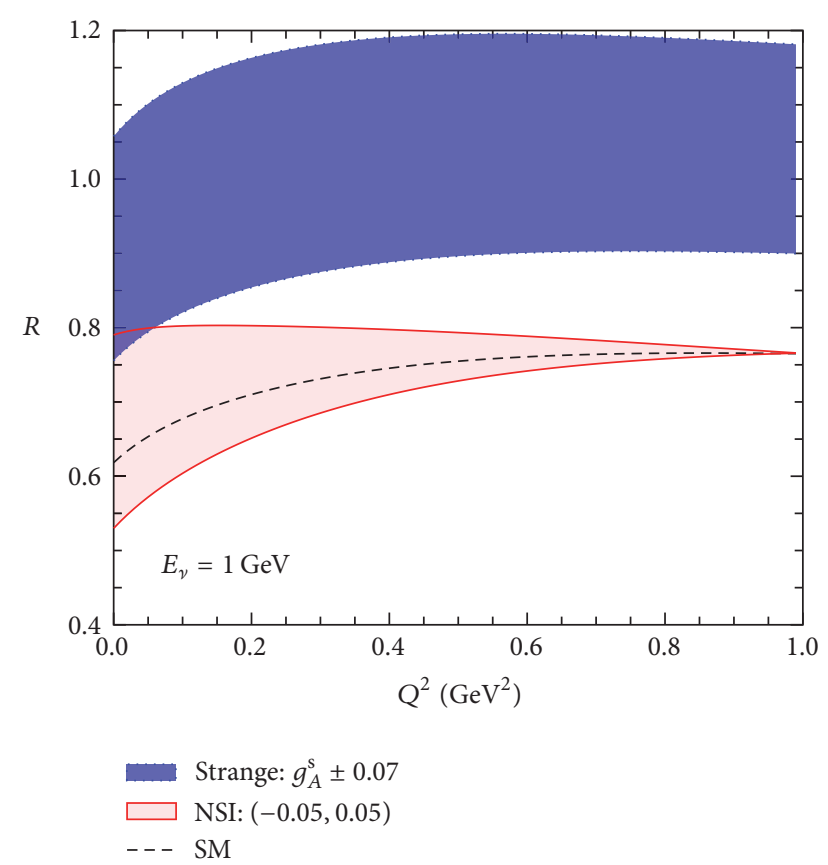

FIGURE 4: Momentum variation of the cross sections ratio for SM, strange quark, and NSI neutrino-nucleon scattering.

Pauli blocking effect on the final nucleons in a local density approximation [62] and is given by the expression [29]

$$
S\left(Q^{2}\right)=1-\frac{D\left(Q^{2}\right)}{N}
$$

where $N$ is the number of neutrons of the nuclear target. Assuming a carbon target, ${ }^{12} \mathrm{C}, D\left(Q^{2}\right)$ takes the form

$$
\begin{aligned}
& D\left(Q^{2}\right) \\
& \quad= \begin{cases}\frac{A}{2}\left[1-\frac{3}{4} \frac{|\mathbf{q}|}{p_{F}}+\frac{1}{24}\left(\frac{|\mathbf{q}|}{p_{F}}\right)^{3}\right], & |\mathbf{q}|<2 p_{F}, \\
0, & |\mathbf{q}|>2 p_{F},\end{cases}
\end{aligned}
$$

with the Fermi momentum $p_{F}=0.220 \mathrm{GeV}$ (for its definition, see $[61,65])$ and $|\mathbf{q}|$ being the magnitude of the three-momentum transfer. Within this framework, the total cross section may be evaluated through numerical integration of the differential cross section (7) as

$$
\sigma\left(E_{\nu}\right)=\int_{0}^{\mathrm{Q}_{\max }^{2}\left(E_{\nu}\right)} S\left(Q^{2}\right) \frac{d \sigma}{d Q^{2}} d Q^{2} .
$$

For NCE scattering, the kinematics of the process provide the approximate upper limit of the momentum transfer $Q^{2}$, as

$$
Q_{\max }^{2}\left(E_{\nu}\right)=\frac{4 m_{\mathcal{N}} E_{\nu}^{2}}{m_{\mathcal{N}}+2 E_{\nu}} .
$$

The obtained results are demonstrated in Figure 5 for protons and in Figure 6 for neutrons for the case of (i) free nucleon scattering, that is, on a hydrogen atom (a), and (ii) medium scattering, that is, for bound nucleons within a carbon atom (b). As expected, for both SM and NSI, the obtained integrated cross section for the $\nu n$ process is much larger than that of the $v p$ reaction. Apparently, one also notices that 

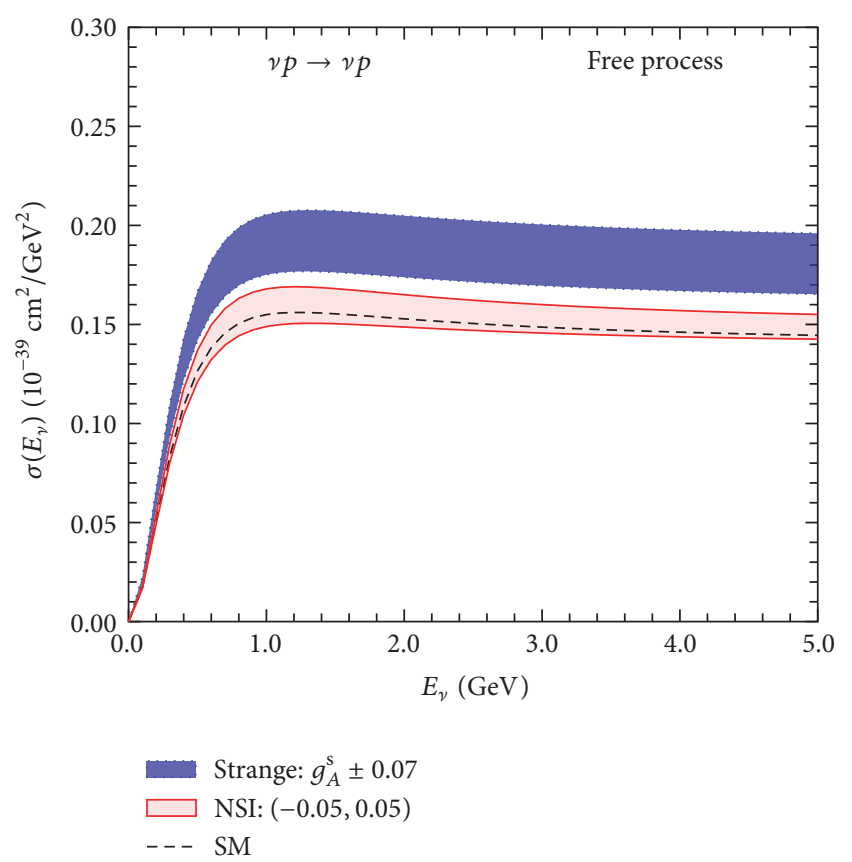

(a)

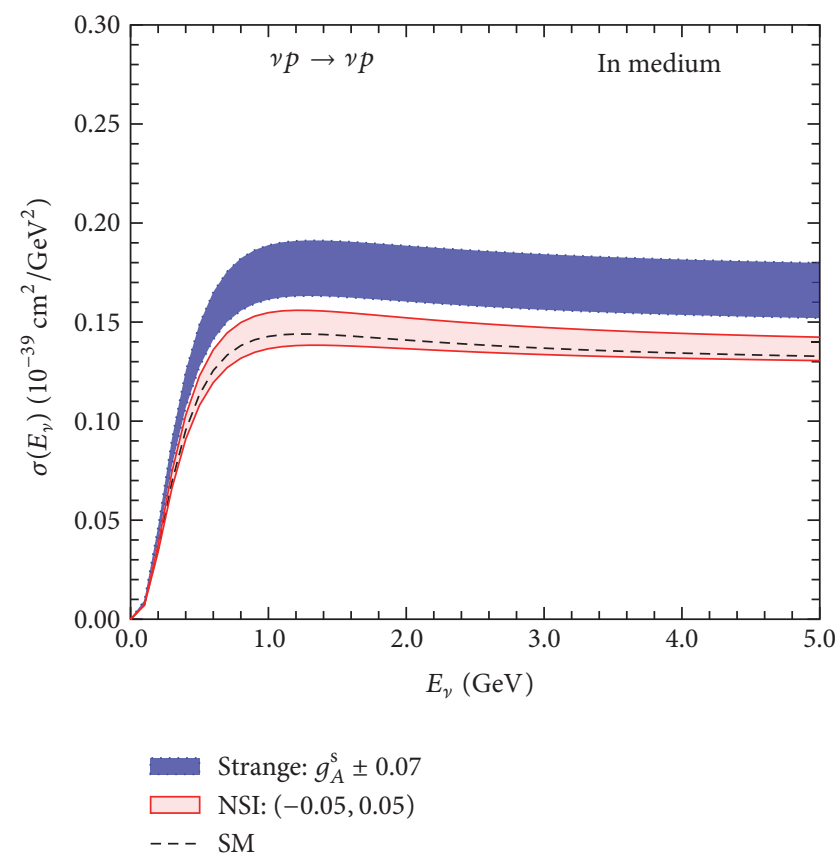

(b)

Figure 5: Total integrated $\nu p \rightarrow v p$ scattering cross section as a function of the incoming neutrino energy due to SM, strange quark, and NSI scattering. The results refer to scattering on free protons (a) and scattering on bound protons, that is, assuming nuclear effects, at a ${ }^{12} \mathrm{C}$ detector (b).

the NCE cross sections, for both $v p \rightarrow v p$ and $v n \rightarrow v n$ scattering channels, are enhanced when potential nonzero NSI are assumed. For the case of neutrino-proton scattering, the considered axial vector strange quark effects dominate the total cross section. On the other hand, focusing on neutrinoneutron scattering, the assumed strangeness of the nucleon leads to suppression of the total cross section. Eventually, we find that the resulting strange quark and NSI bands overlap only for $v n$ processes.

At this point, we find it interesting to focus our discussion on the MiniBooNE experiment and apply the addressed NSI model. Thus, by convoluting the energy distribution of the MiniBooNE neutrino beam with the NSI cross section of (7), we evaluate the flux-integrated differential NSI neutrinonucleon cross sections $\left\langle d \sigma_{p, n} / d T_{p, n}\right\rangle$, through the expression

$$
\begin{aligned}
\left\langle\frac{d \sigma_{p, n}}{d T_{p, n}}\right\rangle= & \int S\left(Q^{2}\right) \frac{d \sigma}{d Q^{2}}\left(E_{v}, Q^{2}\right) \Phi_{\nu}\left(E_{\nu}\right) \\
& \cdot \delta\left(\frac{Q^{2}}{2 m_{\mathcal{N}}}-T_{p, n}\right) d E_{\nu} d Q^{2},
\end{aligned}
$$

where the utilised muon neutrino flux, $\Phi_{\gamma}\left(E_{\gamma}\right)$, is normalised to unity. The results are illustrated in Figure 7 , where it is clearly shown that the nuclear effects become important at low recoil energies. More specifically, for NCE scattering on free nucleons (e.g., for a hydrogen target), the cross section is significantly larger and constantly increasing for low nucleon recoil energies. On the contrary, for the case of bound nucleons within the carbon target material, ${ }^{12} \mathrm{C}$, the behaviour of the cross section changes drastically at low recoil energies and its value minimises for energies $\lesssim 100 \mathrm{MeV}$. As for the total integrated cross sections discussed previously, our results show an overlap between the obtained strange quark and NSI bands only for the processes involving $\nu n \rightarrow v n$ scattering.

We finally test the compatibility of the employed NSI scenario with recent results from the LSND and MiniBooNE experiments. To this aim, concentrating on neutrino-nucleon scattering on a mineral oil $\left(\mathrm{CH}_{2}\right)$, the folded differential cross section reads [47]

$$
\begin{aligned}
\left\langle\frac{d \sigma_{\mathcal{N}}}{d T_{\mathcal{N}}}\right\rangle= & \frac{1}{7}\left\langle\frac{d \sigma_{p}^{H}}{d T_{p}}\right\rangle+\frac{3}{7}\left\langle\frac{d \sigma_{p}^{C}}{d T_{p}}\right\rangle \\
& +\frac{3}{7}\left\langle\frac{d \sigma_{n}^{C}}{d T_{n}}\right\rangle .
\end{aligned}
$$

Then, we evaluate the number of conventional and NSI events as a function of the nucleon recoil energy, $T_{\mathscr{N}}$, by assuming a $\mathrm{CH}_{2}$ detector (i.e., the detector material of the LSND and MiniBooNE experiments) through the expression

$$
N_{\text {events }}=a\left(T_{\mathscr{N}}\right) \int \mathcal{N}_{N} N_{\text {POT }}\left\langle\frac{d \sigma_{\mathcal{N}}}{d T_{\mathscr{N}}}\right\rangle d T_{\mathscr{N}}
$$

In order to confront our present results with the recent MiniBooNE data, we assumed the following experimental quantities: the utilised muon neutrino flux, $\Phi_{\nu}\left(E_{\gamma}\right)$, is normalised to the protons on target (POT) with $N_{\text {POT }}$ denoting 


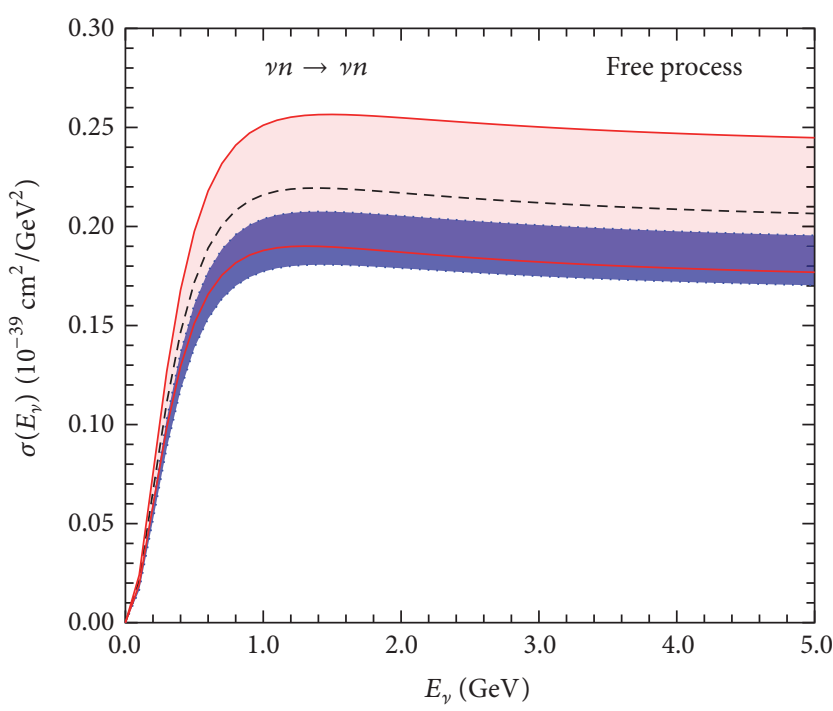

$\square$ Strange: $g_{A}^{\mathrm{s}} \pm 0.07$
$\square$ NSI: $(-0.05,0.05)$
-- SM

(a)

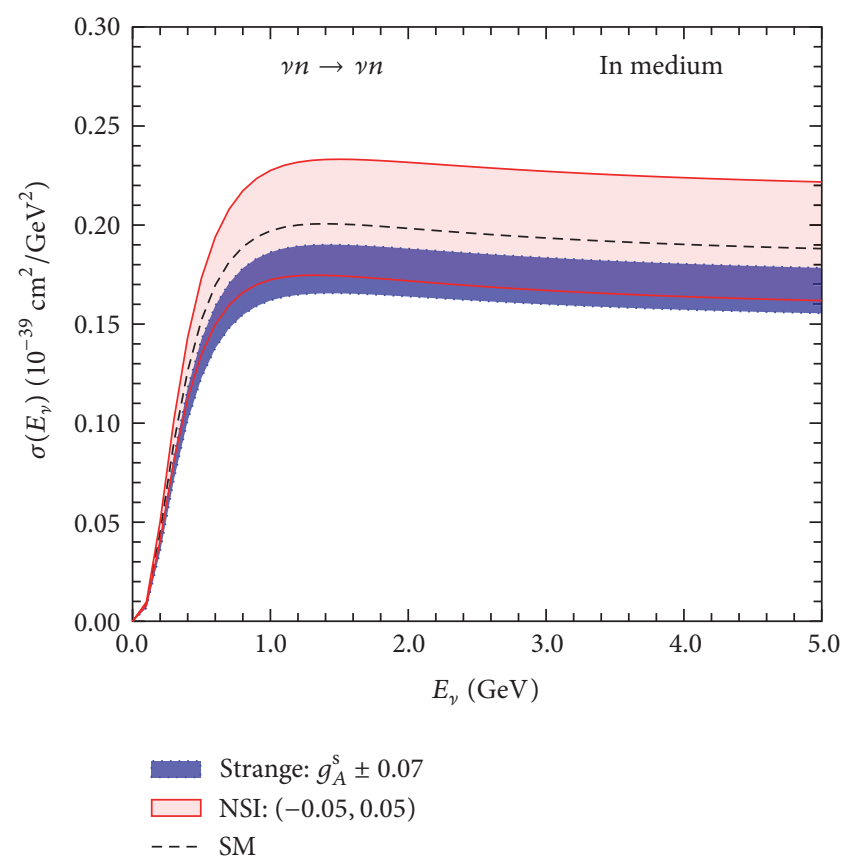

(b)

Figure 6: Same as Figure 5 but for $v n \rightarrow v n$ scattering.
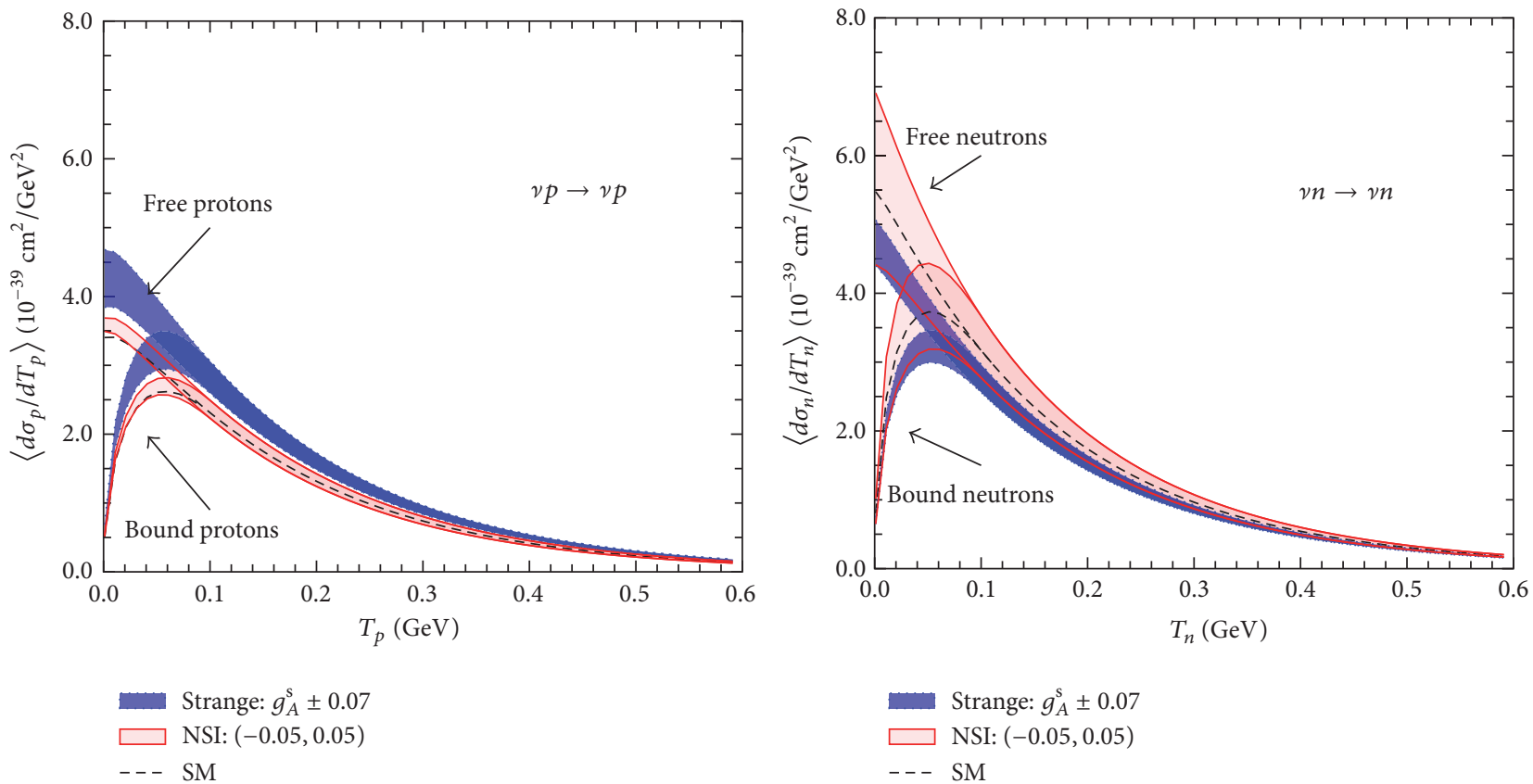

FIGURE 7: Differential cross section as a function of the nucleon (proton or neutron) recoil energy due to SM, strange quark, and NSI. Modifications due to the employed nuclear effects are illustrated and compared with the case of scattering on free nucleons.

the number of POT in the data $[46]$ and $a\left(T_{\mathscr{N}}\right)$ denoting the detector efficiency taken from [49]. The number of nucleon targets in the detector is evaluated as $\mathcal{N}_{N}=N_{A}(4 / 3) \pi R^{3} \rho_{\text {oil }}$, where the density of mineral oil is $\rho_{\text {oil }}=0.845 \mathrm{gr} / \mathrm{cm}^{3}$ at $20^{\circ} \mathrm{C}[48]$ and $N_{A}$ is Avogadro's number. The fiducial volume cut of the detector is adopted from [47]. Figure 8 illustrates the number of events as a function of the nucleon kinetic energy, $T_{\mathscr{N}}$, obtained within the context of the $\mathrm{SM}$, as well as by assuming potential contributions to the rate arising from strange quarks or NSI (for a comparison with the MiniBooNE experimental results, see [47]). The obtained excess of events becomes significant for low recoil energies, reflecting the dipole character of the form factor $G_{D}\left(Q^{2}\right)$ that enters the definition of the NSI nucleon form factors given in (15). 

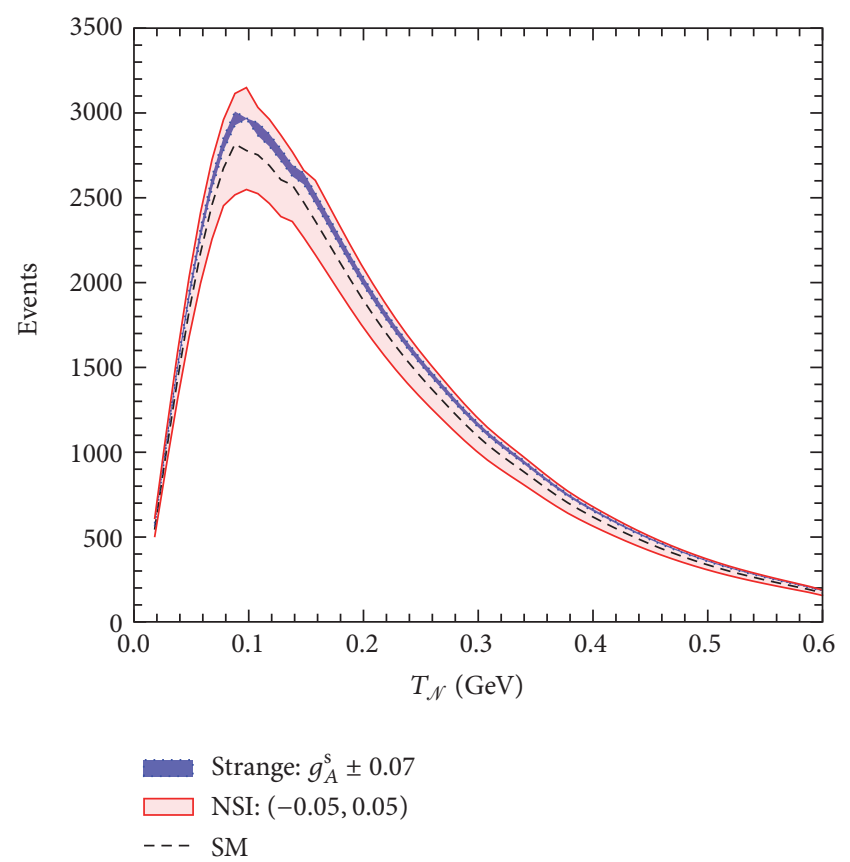

Figure 8: Expected number of events as a function of the nucleon recoil energy $T_{\mathcal{N}}$ assuming contributions due to the SM, strange quark, and NSI.

Motivated by our previous studies, we consider it interesting to estimate the sensitivity of MiniBooNE to nonstandard interactions, through a $\chi^{2}$ fit analysis. By varying one NSI coupling at a time and by neglecting potential strange quark contributions, the minimisation of the $\chi^{2}\left(\epsilon_{\mu}^{u V}\right)$ function provides constraints on the NSI parameters of the order of $\epsilon_{\mu e}^{d V} \approx$ $\epsilon_{\mu e}^{d V}=0.05$. Furthermore, in order to explore the overlap of strange quark and NSI contributions that enter the NCE scattering cross section of (7), a two-parameter combined analysis is performed. By simultaneously varying the strange quark $g_{A}^{\mathrm{s}}$ and NSI $\epsilon_{\mu e}^{u V}$ (setting $\epsilon_{\mu e}^{d V}=0$ ) parameters, the minimisation of $\chi^{2}\left(\epsilon_{\mu e}^{u V}, \Delta s\right)$ yields the contours in the parameter space $\left(\epsilon_{\mu e}^{u V}-\Delta s\right)$ shown in Figure 9, at 68\%, 90\%, and $99 \%$ CL. These results indicate strongly that current neutrinonucleon experiments are favourable facilities to provide new insights and to put severe bounds on nonstandard interaction parameters.

\section{Summary and Conclusions}

In the present work, focusing on the NCE neutrino-nucleon scattering, potential corrections to the SM cross sections that arise from strange quark contributions and nonstandard neutrino-nucleon interactions are comprehensively investigated. In this context, the possibility of probing the relevant model parameters is explored. Furthermore, special effort has been devoted towards exploring the overlap of possible contributions due to strange quarks and NSI. The study involves reliable calculations of the differential and total neutrino-nucleon cross sections by taking into account

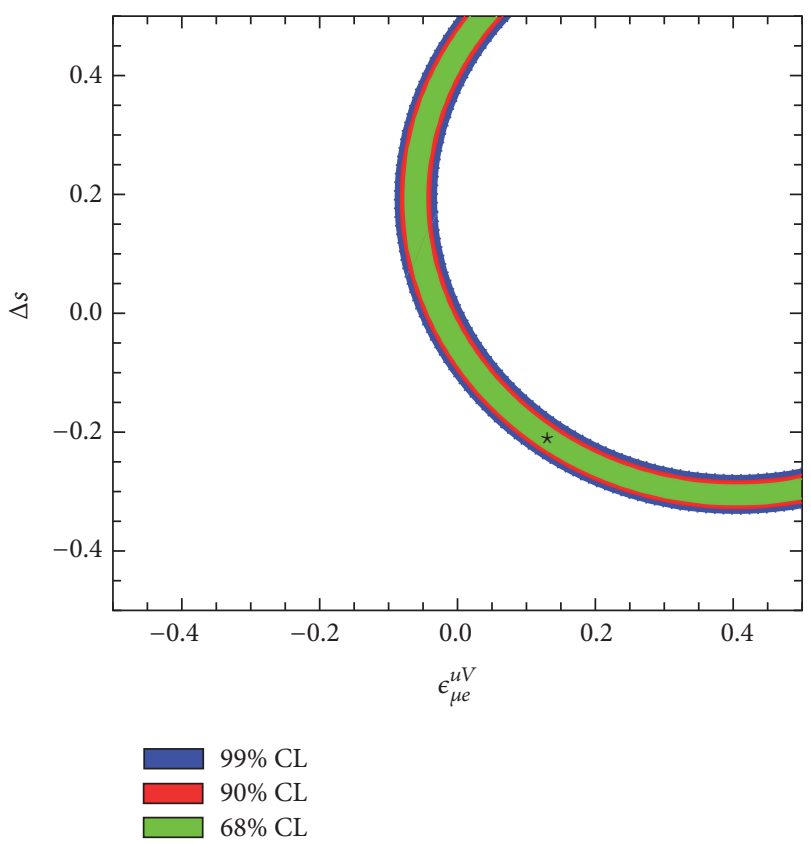

FIGURE 9: Allowed region in the $\left(\epsilon_{\mu e}^{u V}-\Delta s\right)$ plane at MiniBooNE. The best fit point is shown by $\star$.

important nuclear effects such as the Pauli blocking. Within this framework, the NSI contributions originate from the respective nucleon form factors and adopt dipole momentum dependence, while the corresponding cross sections are rather sensitive to the magnitude of the NSI. The latter have a significant impact on the expected number of NCE neutrino-nucleon events and lead to an enhancement of the rate, which may be detectable by the relevant experiments (e.g., MiniBooNE), even for small values of the flavour changing parameters. It is furthermore shown that possible measurements of the ratio of the NSI cross sections for the $\nu p$ process over the $\nu p$ one offer a unique research path to probe NSI.

We stress, however, that the above results refer to forward NSI scattering and thus they do not reproduce accurately the reported MiniBooNE anomaly where an isotropic excess of events was found coming either from electrons or from converted photons. In addition, the NSI contribution would be small if the "standard" value of possible NSI contributions is chosen. Moreover, in this case, the recoiling protons within the mineral oil are likely to have velocity below the Cherenkov threshold and therefore cannot reproduce the Cherenkov ring. On the other hand, the presence of potential nonstandard neutrino-nucleon events may be compatible with the LSND anomaly which did not rely on Cherenkov radiation.

\section{Competing Interests}

The authors declare that there are no competing interests regarding the publication of this paper. 


\section{Acknowledgments}

The authors are grateful to Professor S. N. Gninenko for stimulating discussions.

\section{References}

[1] W. M. Alberico, S. M. Bilenky, and C. Maieron, "Strangeness in the nucleon: neutrino-nucleon and polarized electronnucleon scattering," Physics Reports, vol. 358, no. 4, pp. 227-308, 2002.

[2] J. G. Morfín, J. Nieves, and J. T. Sobczyk, "Recent developments in neutrino/antineutrino-nucleus interactions," Advances in High Energy Physics, vol. 2012, Article ID 934597, 2012.

[3] K. Abe, Y. Hayato, T. Iida et al., "Solar neutrino results in SuperKamiokande-III," Physical Review D, vol. 83, no. 5, Article ID 052010, 19 pages, 2011.

[4] T. Smponias and O. T. Kosmas, "High energy neutrino emission from astrophysical jets in the Galaxy," Advances in High Energy Physics, vol. 2015, Article ID 921757, 7 pages, 2015.

[5] K. G. Balasi, K. Langanke, and G. Martínez-Pinedo, "Neutrinonucleus reactions and their role for supernova dynamics and nucleosynthesis," Progress in Particle and Nuclear Physics, vol. 85, pp. 33-81, 2015.

[6] J. W. F. Valle and J. C. Romao, "Neutrinos in high energy and astroparticle physics," in Physics Textbook, Wiley-VCH, Weinheim, Germany, 2015.

[7] T. S. Kosmas and E. Oset, "Charged current neutrino nucleus reaction cross-sections at intermediate-energies," Physical Review C, vol. 53, no. 3, pp. 1409-1415, 1996.

[8] D. Akimov, P. An, C. Awe et al., "The COHERENT experiment at the spallation neutron source," https://arxiv.org/abs/1509. 08702 .

[9] S. Kerman, V. Sharma, M. Deniz et al., "Coherency in neutrinonucleus elastic scattering," Physical Review D, vol. 93, no. 11, Article ID 113006, 2016.

[10] V. Belov, V. Brudanin, V. Egorov et al., "The $\nu \mathrm{GeN}$ experiment at the Kalinin Nuclear Power Plant," Journal of Instrumentation, vol. 10, no. 12, p. P12011, 2015.

[11] A. G. Beda, V. B. Brudanin, V. G. Egorov et al., "Gemma experiment: the results of neutrino magnetic moment search," Physics of Particles and Nuclei Letters, vol. 10, no. 2, pp. 139-143, 2013.

[12] G. Fernandez Moroni, J. Estrada, E. E. Paolini, G. Cancelo, J. Tiffenberg, and J. Molina, "Charge coupled devices for detection of coherent neutrino-nucleus scattering," Physical Review D, vol. 91, no. 7, Article ID 072001, 2015.

[13] A. Aguilar-Arevalo, X. Bertou, C. Bonifazi et al., "Results of the engineering run of the Coherent Neutrino Nucleus Interaction Experiment (CONNIE)," Journal of Instrumentation, vol. 11, no. 7, Article ID P07024, 2016.

[14] J. Schechter and J. W. F. Valle, "Majorana neutrinos and magnetic fields," Physical Review D, vol. 24, pp. 1883-1889, 1981, Erratum: Physical Review D, vol. 25, article 283, 1982.

[15] T. S. Kosmas and J. D. Vergados, "Sum rules for the inclusive $\mu$ e conversion exotic reaction," Physics Letters B, vol. 217, no. 1-2, pp. 19-24, 1989.

[16] T. S. Kosmas, O. G. Miranda, D. K. Papoulias, M. Tórtola, and J. W. F. Valle, "Probing neutrino magnetic moments at the Spallation Neutron Source facility," Physical Review D, vol. 92, no. 1, Article ID 013011, 2015.
[17] T. S. Kosmas, O. G. Miranda, D. K. Papoulias, M. Tórtola, and J. W. F. Valle, "Sensitivities to neutrino electromagnetic properties at the TEXONO experiment," Physics Letters, Section B: Nuclear, Elementary Particle and High-Energy Physics, vol. 750, pp. 459465, 2015.

[18] S. M. Boucenna, S. Morisi, and J. W. F. Valle, "The low-scale approach to neutrino masses," Advances in High Energy Physics, vol. 2014, Article ID 831598, 15 pages, 2014.

[19] D. V. Forero, M. Tórtola, and J. W. F. Valle, "Global status of neutrino oscillation parameters after Neutrino-2012," Physical Review D, vol. 86, no. 7, Article ID 073012, 2012.

[20] D. Forero, M. Tórtola, and J. Valle, "Neutrino oscillations refitted," Physical Review D, vol. 90, no. 9, 2014.

[21] R. E. Shrock, "Electromagnetic properties and decays of Dirac and Majorana neutrinos in a general class of gauge theories," Nuclear Physics, Section B, vol. 206, no. 3, pp. 359-379, 1982.

[22] B. Kayser, "Majorana neutrinos and their electromagnetic properties," Physical Review D, vol. 26, no. 7, pp. 1662-1670, 1982.

[23] J. F. Nieves, "Electromagnetic properties of Majorana neutrinos," Physical Review D, vol. 26, no. 11, pp. 3152-3158, 1982.

[24] J. F. Beacom and P. Vogel, "Neutrino magnetic moments, flavor mixing, and the super-kamiokande solar data," Physical Review Letters, vol. 83, no. 25-20, article 5222, 1999.

[25] C. Broggini, C. Giunti, and A. Studenikin, "Electromagnetic properties of neutrinos," Advances in High Energy Physics, vol. 2012, Article ID 459526, 47 pages, 2012.

[26] K. G. Balasi, E. Ydrefors, and T. S. Kosmas, “Theoretical study of neutrino scattering off the stable even Mo isotopes at low and intermediate energies," Nuclear Physics A, vol. 868-869, no. 1, pp. 82-98, 2011.

[27] V. Tsakstara and T. S. Kosmas, "Low-energy neutral-current neutrino scattering on Te128,130 isotopes," Physical Review C Nuclear Physics, vol. 83, no. 5, Article ID 054612, 2011.

[28] E. Ydrefors, K. G. Balasi, T. S. Kosmas, and J. Suhonen, "Detailed study of the neutral-current neutrino-nucleus scattering off the stable Mo isotopes," Nuclear Physics A, vol. 896, pp. 1-23, 2012.

[29] C. H. Llewellyn Smith, "Neutrino reactions at accelerator energies," Physics Reports, vol. 3, no. 5, pp. 261-379, 1972.

[30] D. Cline, A. Entenberg, W. Kozanecki et al., "Observation of elastic neutrino-proton scattering," Physical Review Letters, vol. 37, pp. 252-255, 1976.

[31] A. Entenberg, J. Horstkotte, W. Kozanecki et al., "Measurement of the elastic scattering of neutrinos and antineutrinos by protons," Physical Review Letters, vol. 42, no. 18, pp. 1198-1201, 1979.

[32] L. A. Ahrens, S. H. Aronson, P. L. Connolly et al., "Measurement of neutrino-proton and anti-neutrino-proton elastic scattering," Physical Review D, vol. 35, no. 3, pp. 785-809, 1987.

[33] R. A. Smith and E. J. Moniz, "Neutrino reactions on nuclear targets," Nuclear Physics B, vol. 43, pp. 605-622, 1972, Erratum: Nuclear Physics B, vol. 101, pp. 547, 1975.

[34] C. J. Horowitz, H. Kim, D. P. Murdock, and S. Pollock, "Neutrino-nucleus quasifree neutral current reactions and the nucleon strange quark content," Physical Review C, vol. 48, no. 6, pp. 3078-3087, 1993.

[35] M. B. Barbaro, A. De Pace, T. W. Donnelly, A. Molinari, and M. J. Musolf, "Probing nucleon strangeness with neutrinos: nuclear model dependences," Physical Review C-Nuclear Physics, vol. 54, no. 4, pp. 1954-1969, 1996.

[36] W. M. Alberico, M. B. Barbaro, S. M. Bilenky et al., "Inelastic neutrino and anti-neutrino scattering on nuclei and 
'strangeness' of the nucleon,' Nuclear Physics A, vol. 623, pp. 471-497, 1997.

[37] W. M. Alberico, M. B. Barbaro, S. M. Bilenky et al., "The ratio of $\mathrm{p}$ and $\mathrm{n}$ yields in $\mathrm{NC}$ neutrino (anti-neutrino) nucleus scattering and strange form-factors of the nucleon," Physics Letters B, vol. 438, no. 1-2, pp. 9-13, 1998.

[38] F. J. Ernst, R. G. Sachs, and K. C. Wali, "Electromagnetic form factors of the nucleon," Physical Review, vol. 119, no. 3, pp. 1105$1114,1960$.

[39] W. M. Alberico, S. M. Bilenky, C. Giunti, and K. M. Graczyk, "Electromagnetic form factors of the nucleon: new fit and analysis of uncertainties," Physical Review C, vol. 79, no. 6, Article ID 065204, 2009.

[40] G. T. Garvey, S. Krewald, E. Kolbe, and K. Langanke, "Strange quark contributions to neutrino induced quasielastic scattering," Physics Letters B, vol. 289, no. 3-4, pp. 249-254, 1992.

[41] G. T. Garvey, W. C. Louis, and D. H. White, "Determination of proton strange form-factors from neutrino p elastic scattering," Physical Review C, vol. 48, no. 2, p. 761, 1993.

[42] G. Garvey, E. Kolbe, K. Langanke, and S. Krewald, "Role of strange quarks in quasielastic neutrino scattering," Physical Review C, vol. 48, no. 4, pp. 1919-1925, 1993.

[43] A. Acha, K. A. Aniol, D. S. Armstrong et al., "Precision measurements of the nucleon strange form factors at $\mathrm{Q}^{* *} 2 \sim 0.1$ $\mathrm{GeV}^{* *}$ 2," Physical Review Letters, vol. 98, Article ID 032301, 2007.

[44] C. Athanassopoulos, R. L. Burman, L. B. Auerbach, and S. Yellin, "Evidence for $\bar{v}_{\mu} \rightarrow \bar{v}_{e}$ Oscillations from the LSND experiment at the los alamos meson physics facility," Physical Review Letters, vol. 77, no. 15, pp. 3082-3085, 1996.

[45] A. A. Aguilar-Arevalo, C. E. Anderson, A. O. Bazarko et al., "Unexplained excess of electronlike events from a $1-\mathrm{GeV}$ neutrino beam," Physical Review Letters, vol. 102, no. 10, Article ID 101802, 2009.

[46] A. A. Aguilar-Arevalo, C. E. Anderson, A. O. Bazarko et al., "First measurement of the muon neutrino charged current quasielastic double differential cross section," Physical Review D, vol. 81, no. 9, Article ID 092005, 2010.

[47] A. A. Aguilar-Arevalo, C. Anderson, A. Bazarko et al., "Measurement of the neutrino neutral-current elastic differential cross section on mineral oil at $E_{v} \sim 1 \mathrm{GeV}$,' Physical Review D, vol. 82, no. 9, Article ID 092005, 2010.

[48] A. A. Aguilar-Arevalo, B. C. Brown, L. Bugel et al., "First measurement of the muon antineutrino double-differential charged-current quasielastic cross section," Physical Review D, vol. 88, no. 3, Article ID 032001, 31 pages, 2013.

[49] A. A. Aguilar-Arevalo, B. C. Brown, L. Bugel et al., "Measurement of the antineutrino neutral-current elastic differential cross section," Physical Review D, vol. 91, no. 1, Article ID 012004, 12 pages, 2015.

[50] B. Bhattacharya, R. J. Hill, and G. Paz, "Model-independent determination of the axial mass parameter in quasielastic neutrino-nucleon scattering," Physical Review D-Particles, Fields, Gravitation and Cosmology, vol. 84, no. 7, Article ID 073006, 2011.

[51] J. Nieves, J. E. Amaro, and M. Valverde, "Inclusive quasi-elastic neutrino reactions," Physical Review C, vol. 70, no. 5, Article ID 055503, 2004, Erratum: Physical Review C, vol. 72, Article ID 019902, 2005.

[52] J. Nieves, M. Valverde, and M. J. Vicente Vacas, "Inclusive nucleon emission induced by quasielastic neutrino-nucleus interactions," Physical Review C, vol. 73, no. 2, Article ID 025504, 2006.

[53] T. Golan, C. Juszczak, and J. T. Sobczyk, "Effects of final-state interactions in neutrino-nucleus interactions," Physical Review C-Nuclear Physics, vol. 86, no. 1, Article ID 015505, 2012.

[54] J. Nieves, I. Ruiz Simo, and M. J. Vicente Vacas, "The nucleon axial mass and the MiniBooNE quasielastic neutrino-nucleus scattering problem," Physics Letters B, vol. 707, no. 1, pp. 72-75, 2012.

[55] J. Nieves, I. R. Simo, and M. J. V. Vacas, "Inclusive chargedcurrent neutrino-nucleus reactions," Physical Review C, vol. 83, no. 4, Article ID 045501, 2011.

[56] S. N. Gninenko, "MiniBooNE anomaly and heavy neutrino decay," Physical Review Letters, vol. 103, no. 24, Article ID 241802, 2009.

[57] S. N. Gninenko, "Resolution of puzzles from the LSND, KARMEN, and MiniBooNE experiments," Physical Review D, vol. 83, no. 1, Article ID 015015, 2011.

[58] S. N. Gninenko, "New limits on radiative sterile neutrino decays from a search for single photons in neutrino interactions," Physics Letters B, vol. 710, no. 1, pp. 86-90, 2012.

[59] T. S. Kosmas and J. D. Vergados, "Nuclear densities with fractional occupation probabilities of the states," Nuclear Physics A, vol. 536, no. 1, pp. 72-86, 1992.

[60] Y. Umino, J. M. Udias, and P. J. Mulders, "Exchange current corrections to neutrino-nucleus scattering," Physical Review Letters, vol. 74, no. 25, pp. 4993-4996, 1995.

[61] T. Leitner, O. Buss, L. Alvarez-Ruso, and U. Mosel, "Electronand neutrino-nucleus scattering from the quasielastic to the resonance region," Physical Review C, vol. 79, no. 3, Article ID 034601, 2009.

[62] T. Leitner, L. Alvarez-Ruso, and U. Mosel, "Neutral current neutrino-nucleus interactions at intermediate energies," Physical Review C, vol. 74, no. 6, Article ID 065502, 2006.

[63] P. G. Giannaka and T. S. Kosmas, "Electron capture cross sections for stellar nucleosynthesis," Advances in High Energy Physics, vol. 2015, Article ID 398796, 11 pages, 2015.

[64] P. G. Giannaka and T. S. Kosmas, "Detailed description of exclusive muon capture rates using realistic two-body forces," Physical Review C, vol. 92, no. 1, Article ID 014606, 2015.

[65] T. Leitner, L. Alvarez-Ruso, and U. Mosel, "Charged current neutrino-nucleus interactions at intermediate energies," Physical Review C, vol. 73, no. 6, Article ID 065502, 2006.

[66] D. K. Papoulias and T. S. Kosmas, "Nuclear aspects of neutral current non-standard $\nu$-nucleus reactions and the role of the exotic $\mu^{-} \rightarrow \mathrm{e}^{-}$transitions experimental limits," Physics Letters, Section B: Nuclear, Elementary Particle and High-Energy Physics, vol. 728, pp. 482-488, 2014.

[67] D. K. Papoulias and T. S. Kosmas, "Standard and nonstandard neutrino-nucleus reactions cross sections and event rates to neutrino detection experiments," Advances in High Energy Physics, vol. 2015, Article ID 763648, 17 pages, 2015.

[68] D. K. Papoulias and T. S. Kosmas, "Neutrino transition magnetic moments within the non-standard neutrino-nucleus interactions," Physics Letters B, vol. 747, pp. 454-459, 2015.

[69] V. Barger, R. J. N. Phillips, and K. Whisnant, "Solar-neutrino solutions with matter-enhanced flavor-changing neutralcurrent scattering," Physical Review D, vol. 44, no. 6, pp. 1629-1643, 1991.

[70] T. Ohlsson, "Status of non-standard neutrino interactions," Reports on Progress in Physics, vol. 76, no. 4, Article ID 044201, 2013. 
[71] O. G. Miranda and H. Nunokawa, "Non standard neutrino interactions: current status and future prospects," New Journal of Physics, vol. 17, no. 9, Article ID 095002, 2015.

[72] A. Rashed, M. Duraisamy, and A. Datta, "Nonstandard interactions of tau neutrino via charged Higgs and $W^{\prime}$ contribution," Physical Review D, vol. 87, no. 1, Article ID 013002, 2013.

[73] A. Rashed, P. Sharma, and A. Datta, "Tau-neutrino as a probe of nonstandard interaction," Nuclear Physics B, vol. 877, no. 3, pp. 662-682, 2013. 

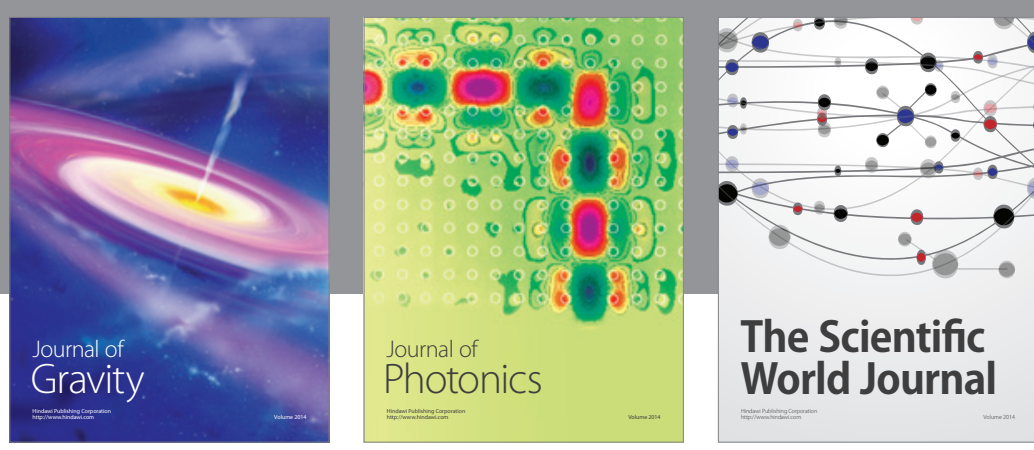

The Scientific World Journal
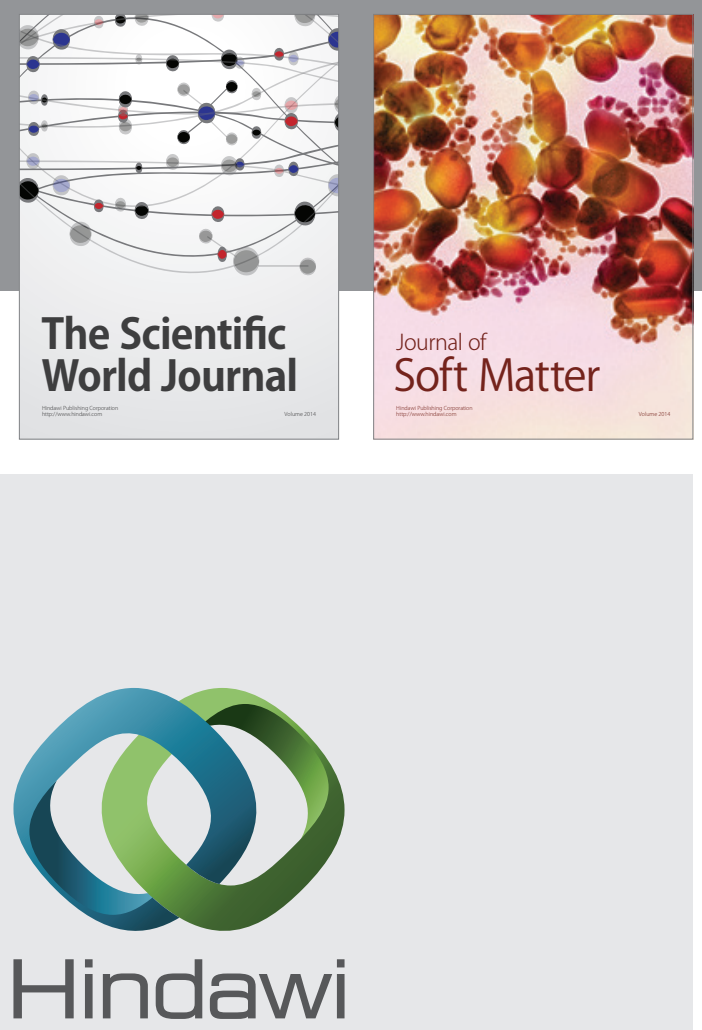

Submit your manuscripts at

http://www.hindawi.com

nternational Journal of

Statistical Mechanics
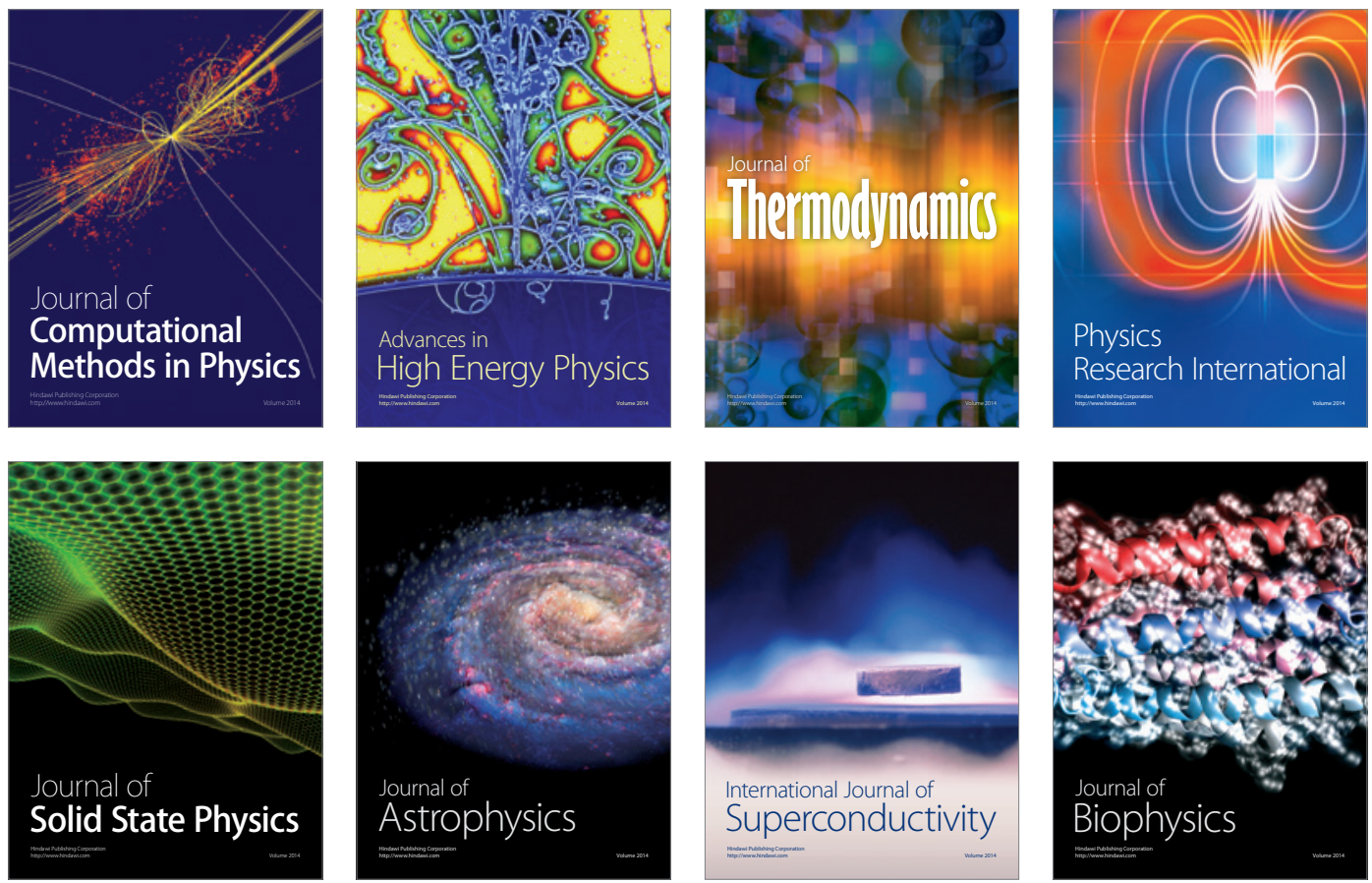
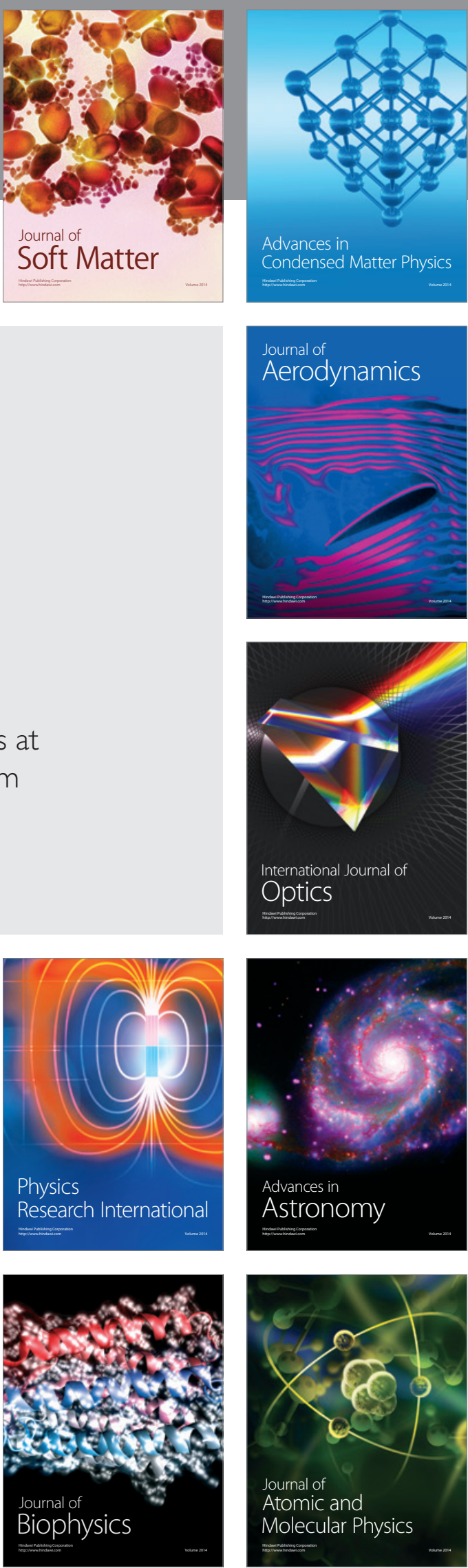